\title{
AC 2010-1956: IMPROVING CONCEPTUAL UNDERSTANDING IN PROBABILITY AND STATISTICS
}

Dean Jensen, South Dakota School of Mines and Technology

Stuart Kellogg, South Dakota School of Mines and Technology 


\title{
Improving Conceptual Understanding in Probability and Statistics
}

\begin{abstract}
Program as well as course assessments indicate that, while technical skills are generally good, a weakness remains in students' ability to translate information and skill sets from one Carnegie unit (one course) to another. The inability to apply probability and/or statistical concepts in different problem contexts is particularly problematic for undergraduate students in industrial engineering. Despite the completion of six credit hours in probability and statistics, undergraduate students continue to have difficulty translating that information to industrial engineering applications in quality control, stochastic models, and work measurements. Historical data using the probability and statistics concepts inventory (Figure 1 below) indicates that, for most students, true conceptual understanding of probability basics remains elusive. This problem may be exacerbated somewhat due to a disconnect between the theory covered in the mathematics department and the applications needed in the industrial engineering program. In this paper we discuss some of the initial inroads towards improving conceptual understanding in the industrial program from a historical perspective and include examples of virtual experiments, technology enabled support modules, and collaborative learning activities. In addition to use of the concepts inventory, program assessments include use of Fundamentals of Engineering (FE) exam, embedded assessments in subsequent courses, and analysis of dwell time and module usage for online support. We conclude with the current status of the initiative and a vision for a collaborative learning approach to statistical concepts through classroom inversion.
\end{abstract}

\section{Introduction}

Statistics is an important element of the curriculum for students in a variety of majors including engineering, business, and the social sciences. Increasingly, elements of data analysis and probability are being emphasized in industry in a variety of disciplines and is becoming increasingly prevalent not only in accreditation requirements but in K-12 standards ${ }^{1}$. For the industrial engineering and the engineering management disciplines, a solid foundation of statistical reasoning is critical. While Fundamentals of Engineering (FE) analysis and course assessments indicate that, in general, student technical skills are good, a weakness remains in students' abilities to translate information and skill sets from one Carnegie unit to another. This is particularly problematic for industrial engineering students who complete 6 credits in probability and statistics, but have difficulty translating that information to industrial engineering applications in simulation, quality control, stochastic models, work measurements, and human factors. Indeed, long term tracking over 6 years utilizing the Fundamentals of Engineering and a Concepts Inventory show little, if any, gains in statistical reasoning (see Figure 1 below). 

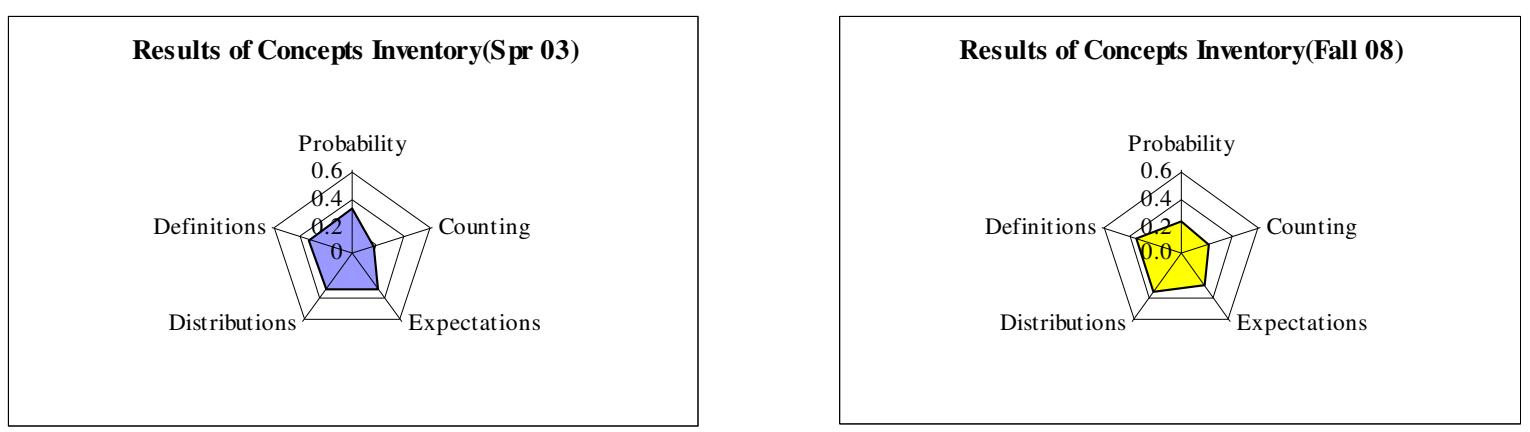

Figure 1. Probability and Statistics Concept Inventory Results (2003, 2008)

Figure 1 shows results from a campus inventory given in the second semester of a probability and statistics sequence. Enrollment in the course is predominantly industrial engineering majors. Figure 1 reflects limited understanding of fundamental concepts but this performance is neither new nor limited to probability and statistics. There is a significant and growing body of research that indicates that students' inability to translate theory to alternative applications may very well rest with students' conceptual understanding or with strongly-held misconceptions ${ }^{2,3}$ within a discipline even after many years of formal and informal training. Several investigations including three video case studies, Lessons from Thin Air, Private Universe, and Can We Believe our Eyes ${ }^{4}$ demonstrate that traditional instruction does little to help students revise their understanding of important concepts. Indeed, the concept inventory movement was spurred by the development and implementation of the Force Concept Inventory ${ }^{5,6}$ as a mechanism to identify student misconceptions when entering a course and check for gains after completing a course. Garfield and Ahlgren ${ }^{7}$ provide an extensive review of students' difficulties learning basic probability and statistics concepts and provided much of the initial framework for development of the Statistics Concept Inventory (SCI) ${ }^{8}$. Additional information on available concept inventories and associated research may be found at the NSF supported Concept Inventory Central website ${ }^{9}$.

\section{A Theoretical Framework for Improving Conceptual Understanding}

Through much of the research associated with concept inventories with repeated testing, it was discovered that students gain the most conceptual knowledge in interactive engagement courses, as opposed to traditional lectures ${ }^{10}$. This is not too surprising and is consistent with the research on pedagogies of engagement ${ }^{11}$ first reported by Karl Smith and colleagues. A new pedagogical technique called model-eliciting activities (MEAs) holds considerable promise for overcoming misconceptions and building a robust foundation for richer engagement with the material and subsequently better conceptual understanding. MEAs were first developed to elicit problemsolving strategies in mathematics, but have since been developed in a number of other disciplines including engineering science ${ }^{12}$.

Before describing the scaffolding approach adopted by the industrial engineering department, we need to provide a short introduction on cognitive development. A fundamental premise of virtually all student development models is that true intellectual growth will not occur unless the foundational skills (conceptual understanding) is firmly grounded. Felder and Brent ${ }^{13}$ provide an excellent summary review of different developmental models. Karlin and Kellogg ${ }^{14}$ have incorporated much of that work along other psycho-social models to provide a conceptual 
framework for holistic learner development (HLD). While the scope of the HLD model is beyond that required for this paper, a fundamental component of the model is the Steps for Better Thinking model proposed by Wolcott and Lynch ${ }^{15}$ and shown below in Figure 2.

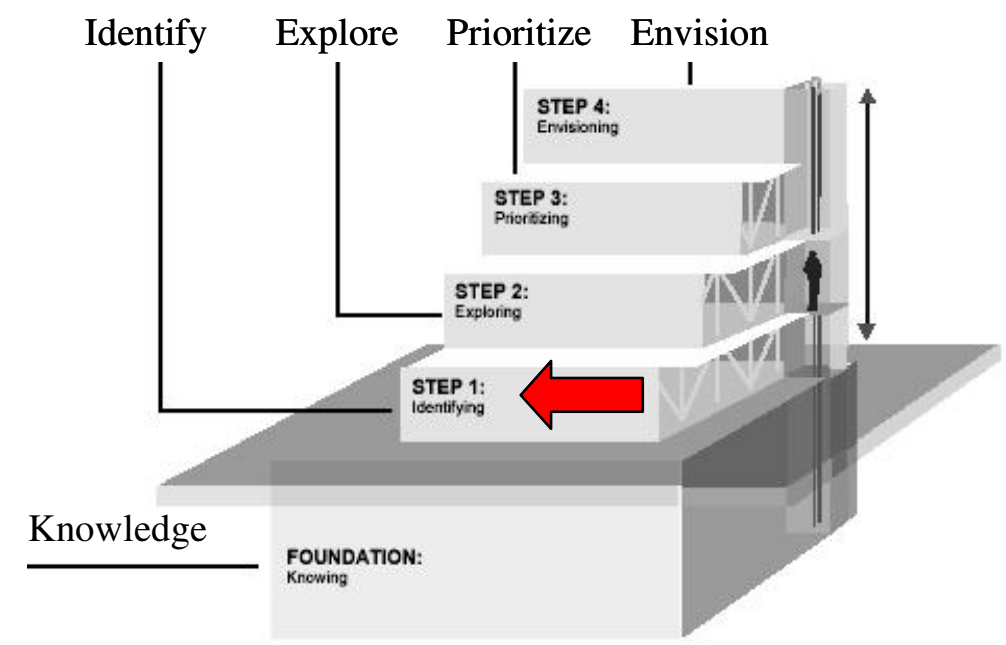

Figure 2. Conceptual Model for Steps for Better Thinking

Conceptual advantages of this model is that it is relatively easy for engineering educators to understand, it has an assessment rubric, and it highlights the need to ensure that students have access to the foundational skills needed for intellectual growth to occur. Stated another way, it is difficult, if not impossible for intellectual gains if students do not fully understand the fundamental concepts of the theory in which they are engaged.

Given this theoretical framework, a logical approach would be to invert the classroom and redesign the probability and statistics courses to incorporate MEAs and real world problem solving activities. That in itself would embody considerable effort, but the problem is further complicated by the fact that the department does not own the required probability and statistics courses. Consequently, we needed to develop an approach that bridged industrial engineering applications in stochastic models, simulation, and quality control with basic theory presented in probability and statistics. We also needed a mechanism to reinforce and integrate foundational skills while simultaneously covering advanced material in other courses. To do this, the department adopted a three pronged approach to improve student conceptual understanding in probability and statistics.

1. Provide a set of technology enabled support modules that support conceptual understanding and alternative modes of intellectual inquiry

2. Reinforce probability concepts through fundamental review and engagement in stochastic models

3. Reinforce statistical concepts through virtual applications in quality control

\section{Support Modules for Probability and Statistics}

The department has developed over 20 interactive support modules for probability and statistics. Modules include coverage of basic probability, distributions, probability plotter/calculator, 
estimators, central limit theorem, differences between the normal and Student's T distributions, hypothesis testing, and the memory-less property of the exponential distribution. Modules are available online and are fully accessible by any computer with internet access. Modules vary in coverage depending on the level required. For example, the module on the central limit theorem covers alternative modes of intellectual inquiry by providing a simple dice example, an experimental sample from a manufacturing process, a heuristic derivation, and a formal derivation using moment generating functions. Most industrial engineering students find the heuristic derivation fairly helpful whereas most non-engineering students enjoy the manufacturing or dice examples. Very few students access the formal derivation even though it is the primary method utilized in most introductory texts. The distribution pages provide summary information, characteristics, relationships to other distributions and a plotter for each distribution and are typically accessed by students in stochastic models and simulation. A sample module frequently accessed by students shows confidence interval differences between the normal and Student's T distributions is shown in Figure 3 below.

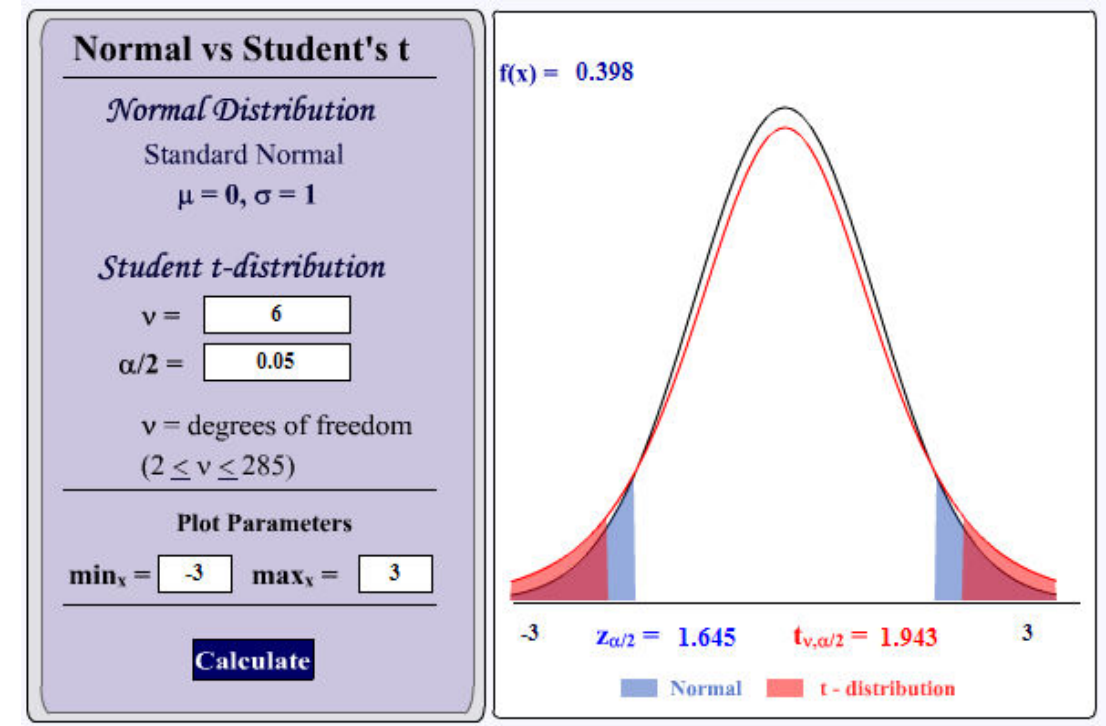

Figure 3. Graphical Display from the Student T vs Normal Distribution Module

Another popular module allows students to explore type I and type II errors as one changes sample sizes and critical values for acceptance or rejection of a lot (see Figure 4). 


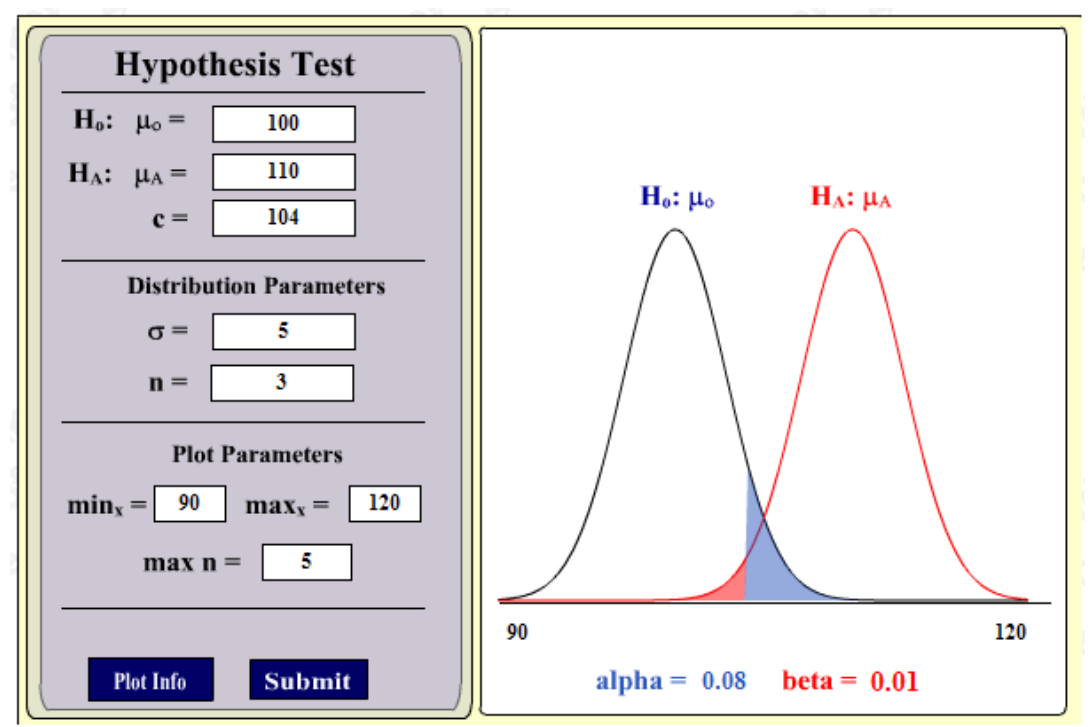

Figure 4. Graphical Display from the Hypothesis Testing Module

Besides principles of good practice in multi-media design ${ }^{16,17}$, a number of important criteria were considered in the development of the review modules. Specifically,

- Where appropriate, modules should consider a variety of learning styles

- Review modules should primarily be focused at knowledge (Foundational) level on the Steps for Better Thinking model (see Figure 2).

- Review modules should be relatively short and should provide opportunities for exploration of applications related to the topic.

- Embedded online interactive exercises should be self-correcting.

- Navigation through review modules should be student-controlled.

- All materials developed should be suitable for incorporation with a classroom project or as a stand-alone review module for asynchronous delivery.

\section{Concept Reinforcement in Stochastic Models}

Stochastic models is highly dependent on students' understanding of basic probability, conditional probability, the exponential family of distributions, and the memory-less property of the exponential distribution and considerable class time is devoted to review of those topics. The syllabus includes a requirement to review those modules immediately prior to class coverage of those topics. Nevertheless, the instructor reinforces these fundamental concepts in class through review and in class group activities. Class activities can include group discussions, game show format review of fundamental theory ${ }^{18}$, or simple in class group calculation activities. As a consequence class progression is slow at first, but gradually picks up as students become more comfortable with the support modules and building connections between basic theory and the more advanced models in stochastic models.

To the extent possible, the course also incorporates a modified model eliciting activity or MEA. More or less, this is just a fancy way of stating that we are essentially neophytes in this area and, because of time and logistical constraints, we have resorted to a less formal approach than a true MEA. Nevertheless, we follow the philosophical construct of open-ended problem solving and model elicitation. A recent example that was fairly popular with the students 
includes data collection on the time between orders of a fruit cup at a popular on-campus coffee bar. Prior to the exercise, students are asked to review the expectation and distribution modules and explore distribution shapes via the plotter/calculator. Students are then given basic information, a timeline for data collection, and detailed information as to how data is to be collected. Following data collection, students are assigned to informal groups in class and asked to a) determine the underlying distribution and b) determine the parameters for the distribution. This is an enormously tough concept for students and initially, groups are slow to respond. Eventually however, one of the groups will propose a histogram or other method as a means of selecting an underlying distribution and deeper engagement with the problem follows shortly. The session is halted ten minutes prior to the end of class so that the instructor may demonstrate the maximum likelihood estimator module. Student groups are then given a formal assignment to determine the best distribution/parameter fit from the available data. The next class session is devoted to a discussion of discrepancies between groups, transference to a more realistic application such as time between arrivals at an emergency room, and finally, the implications distribution selection has for incorporation in a queuing model.

Obviously, these activities take time and there is a trade-off to be made between course content and conceptual understanding. The fundamental principle at stake is whether or not students learn more by covering less. For example, the instructor no longer covers the M/G/1 or models with non-Poisson inputs with the expectation that students now more fully recognize when such models are appropriate and can resort to alternative formulations or simulations when the situation dictates. Course embedded assessments covered in a later section indicate this is a reasonable assumption.

\section{Concept Reinforcement in Quality Control}

The undergraduate quality control course relies upon the students' background with continuous and discrete distributions, hypothesis tests, and conditional probability concepts developed in two prerequisites courses in mathematical statistics. As with the Stochastic Modeling course, approximately $1 / 4$ of the semester is spent reviewing how to apply these prerequisites in an industrial or service application.

One of the first reinforcement activities for this course includes an in-class review exercise for hypothesis testing. This exercise includes a review and reinforcing application practice on hypothesis tests and distribution yield; distribution assumption checks; single sample acceptance plans; and MIL STD 105E acceptance plan operations. The in-class exercise has the students develop a decision-table for selecting the most appropriate hypothesis test for a range of situations. One of the student sample products from this exercise is shown in Figure 5 below. While it is recognized that the graphic Figure 5 is difficult to read, it is an element taken directly from a student journal. Journal entries (such as shown in Figures 5 and 6) are non-graded, but may be used for exam, in-class open-ended problem solving activities, or hand simulations. 


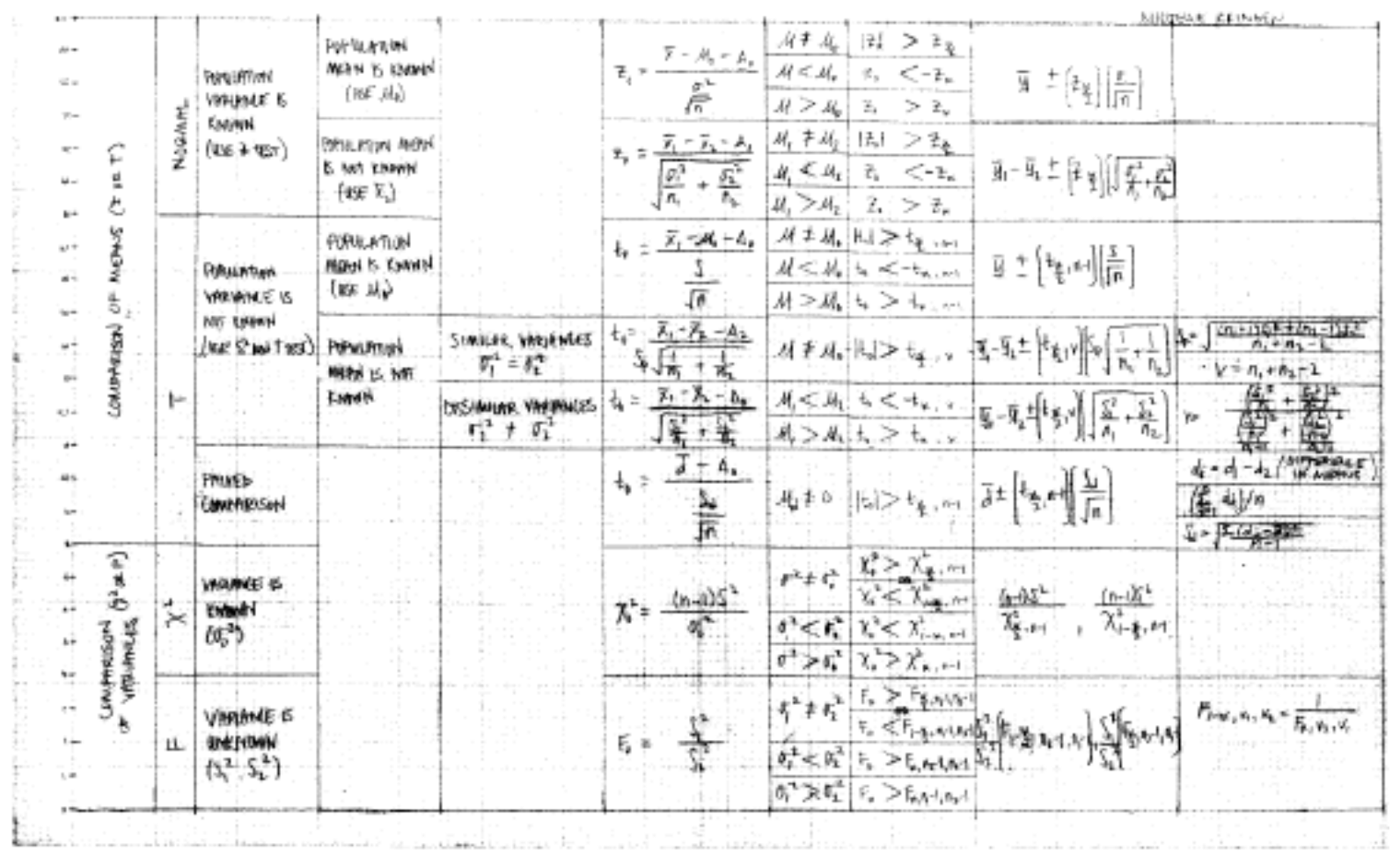

Figure 5. Class-developed decision table for hypothesis testing.

All students in the course must prepare an engineering notebook (individual studentdeveloped reference material) implementation. Essentially, anything the student can write, copy, cut, staple, paste, glue or otherwise persuade to reside permanently within their engineering notebook can be used as a reference on their test(s). The notebooks are the common 9-3/4" x 71/2", 80 - 100 page composition books carried by office supply store chains for about $\$ 2.50$. Students typically paste a subset of lecture slides, reference tables, classroom examples, and student-developed decision trees in the notebooks. An advantage of the journal entry format is that students may take ownership of the format and express difficult concepts through alternate typologies. Figure 5 above, for example, demonstrates a very linear or analytical format, whereas students display information from a more conceptual framework.

In addition, the course incorporates on-line spreadsheet templates for normal probability plots; continuous variables, attributes, individuals control charts; gauge repeatability and reproducibility; and acceptance sampling randomization. These templates allow students to simulate and explore control chart performance under different scenarios. Figure 6 (below) shows screen captures for one such experiment: a comparison of time to signal for Exponentially Weighted Moving Average (top), Moving Average (center), and CUSUM (bottom) control charts. In this example, students conduct virtual experiments using the Excel random number generator and the spreadsheet templates to see how quickly an out-of-control signal is generated for (1) a small shift of $0.75 \sigma,(2)$ a medium shift of $1.5 \sigma$, (3) a large shift of $2.5 \sigma$ and (4) a slow process drift of $1 \%$ increase per unit. (For the large shift and the student's selected parameters, the Moving Average chart outperforms the EWMA and CUSUM charts by at least two sampling periods.) 

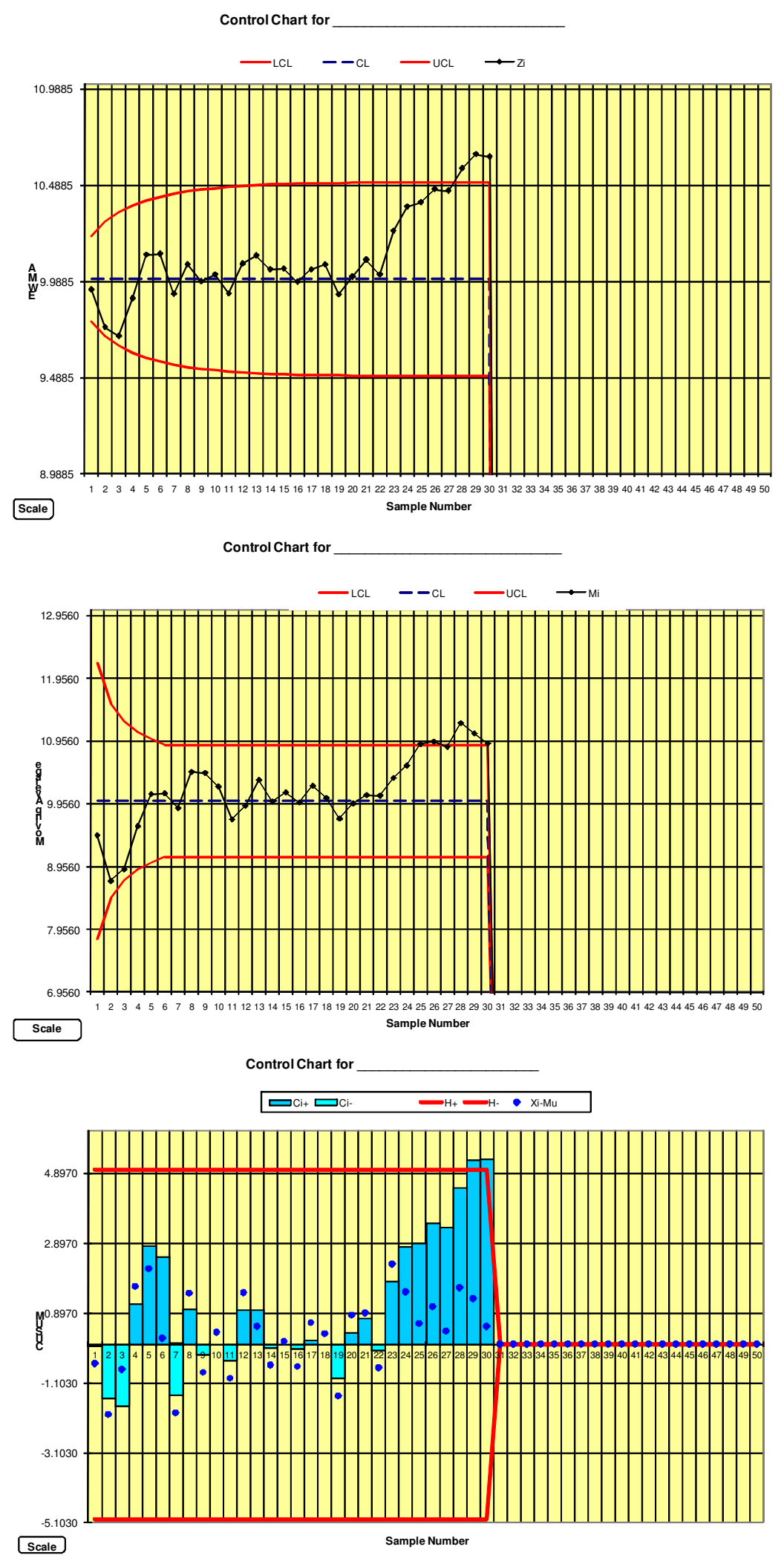

Figure 6. Example of spreadsheet simulation/comparison of control chart performance. 
Much of the course has been redesigned to incorporate hands-on applications of the SQC tools and include applications involving Deming's Red Bead Experiment, Grip Strength Hypothesis Testing, and Caliper and Vernier Micrometer Gauge System Capability Study. Additional hand simulations have been developed to help demonstrate variation and manufacturing and production processes.

\section{Assessment Data}

The department incorporates four different measures to track student conceptual understanding and areas for improvement. These include (1) tracking number of hits and time on task for support modules, (2) embedded assessments in stochastic models, (3) embedded assessments in quality control, and (4) longitudinal tracking via the Statistics Concept Inventory (SCI).

Student access and dwell time for technology enabled support modules are tracked to determine which modules seem to be more effective in terms of support. Figure 7 below shows that students tend to visit the site during peak times of the semester when it is needed for review or when it is needed for a MEA. The StatTracker software captures page loads, unique visitors, and returning visitor data along a user defined time interval for a defined sample size (in our case 100).

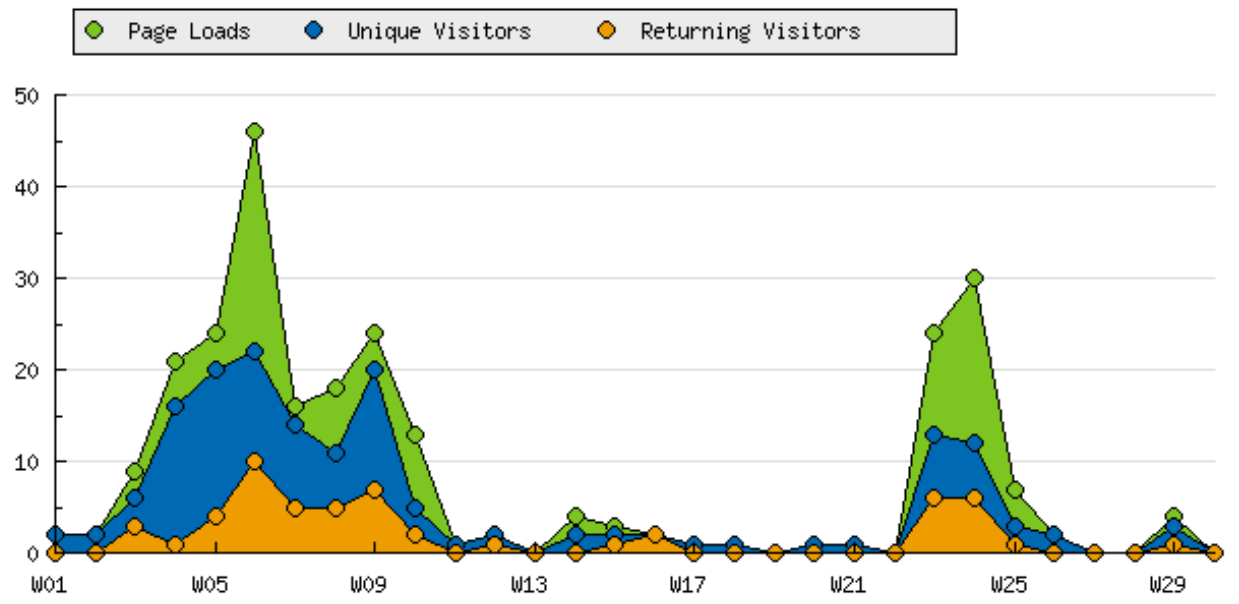

Figure 7. Tracking Student Access to Probability Modules

Of greater value to us is which pages are of primary interest to students and the dwell time at those pages. The probability calculator/plotter, hypothesis test, and distribution modules seem to be of primary interest to students. Dwell time for all probability/statistics modules is shown below in Figure 8. 


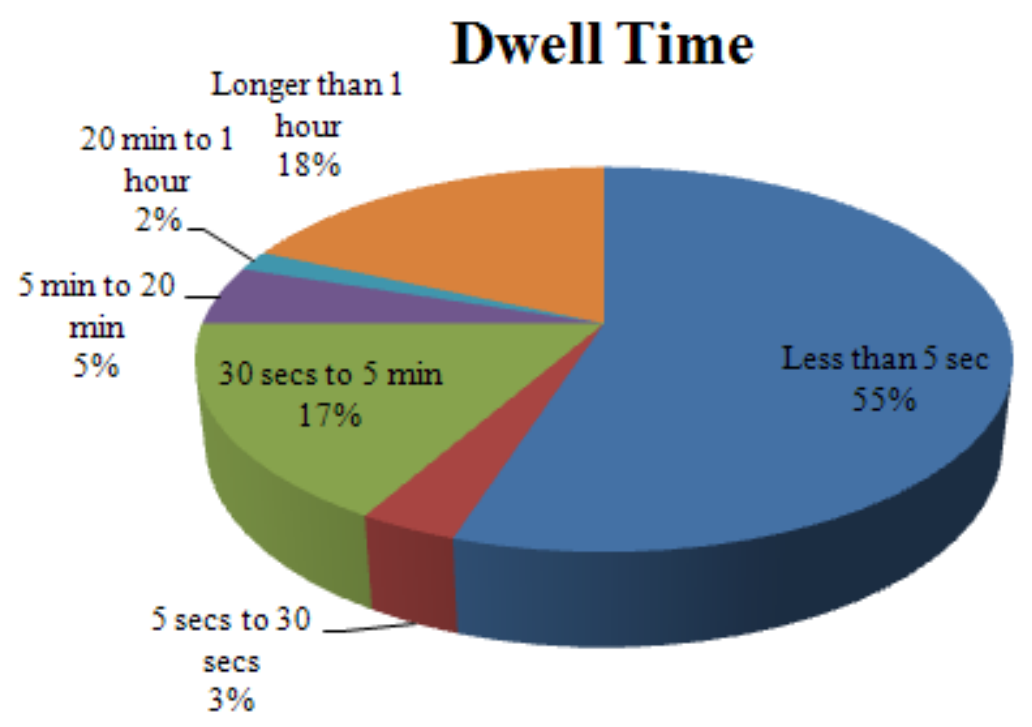

Figure 8. Dwell Time on Probability and Statistics Review Modules

While the bulk of students spend less than 5 seconds on a particular page, it should be remembered that students must navigate to the particular module of interest. Once there, roughly half the students spend 5-20 minutes on a particular module and half spend over 20 minutes. A significant number of students (18.3\%) spend over an hour on some modules.

Embedded assessments are also incorporated in stochastic models and quality control. Embedded assessments in stochastic models are incorporated within homeworks and as short non-graded quiz items that cover concepts in probability, distributions, properties of distributions, the central limit theorem, statistics, and the memory-less property of the exponential. Results over the past five years are summarized in Figure 9 below.

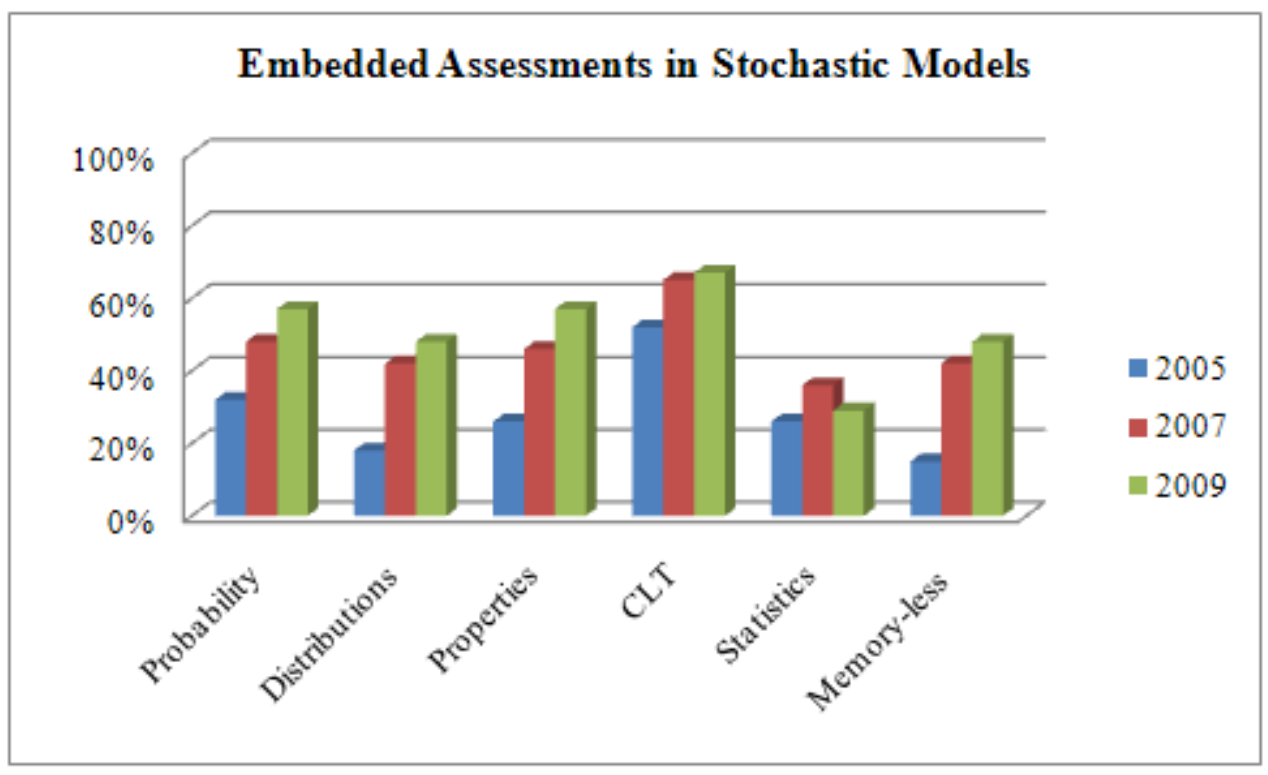

Figure 9. Correct Percentages in Probability and Statics Concepts 
Conceptual gains have been made in all areas and range from an $8.9 \%$ gain in basic statistics to a $78.6 \%$ gain in the memory-less property. Nevertheless, with the possible exception of the central limit theorem, overall gains in conceptual understanding remain low. Even then, statistics scores seem to indicate that students still have difficulty knowing when to apply different sampling distributions and when the central limit allows use of the normal. While it should be noted that statistics is not covered in this course so it is possible scores remain low on embedded assessments here for the simple reason that the course incorporates no meaningful application. That being said, it is entirely possible that student understanding regresses in the areas of probability and distributions once removed from the course for the same reasons.

Assessment of student progress in quality control was broken into three areas: SQC Basics content, covering terms, cumulative density function and hypothesis test applications; Continuous Variables Control Charts, covering basic chart interpretation, terms, x-bar-, R-, scharts, gauge R\&R and process capability analysis; and SQC Individuals Charts and Acceptance Sampling, covering $\mathrm{x}$ - and moving range charts, moving average, EWMA, and CUSUM charts, and single sample acceptance plans, including MIL STD 105E. These data have been tracked over the past five years, and correlated with developed materials (as described below).

For the SQC Basics content areas, the initial assessment (in Fall of 2005) showed a distinct lack of capacity for applying the basic principles to common industrial situations. After discussion with the students, it was discovered that most of them had little applied-practice problem experience. To salvage student course grades (and the instructor's enthusiasm) it was decided to offer an optional, "second chance exam", capping the maximum score at $89 \%$. This exam was continued through the Fall of 2007, and the maximum correct outcome is shown in Figure 10 (below).

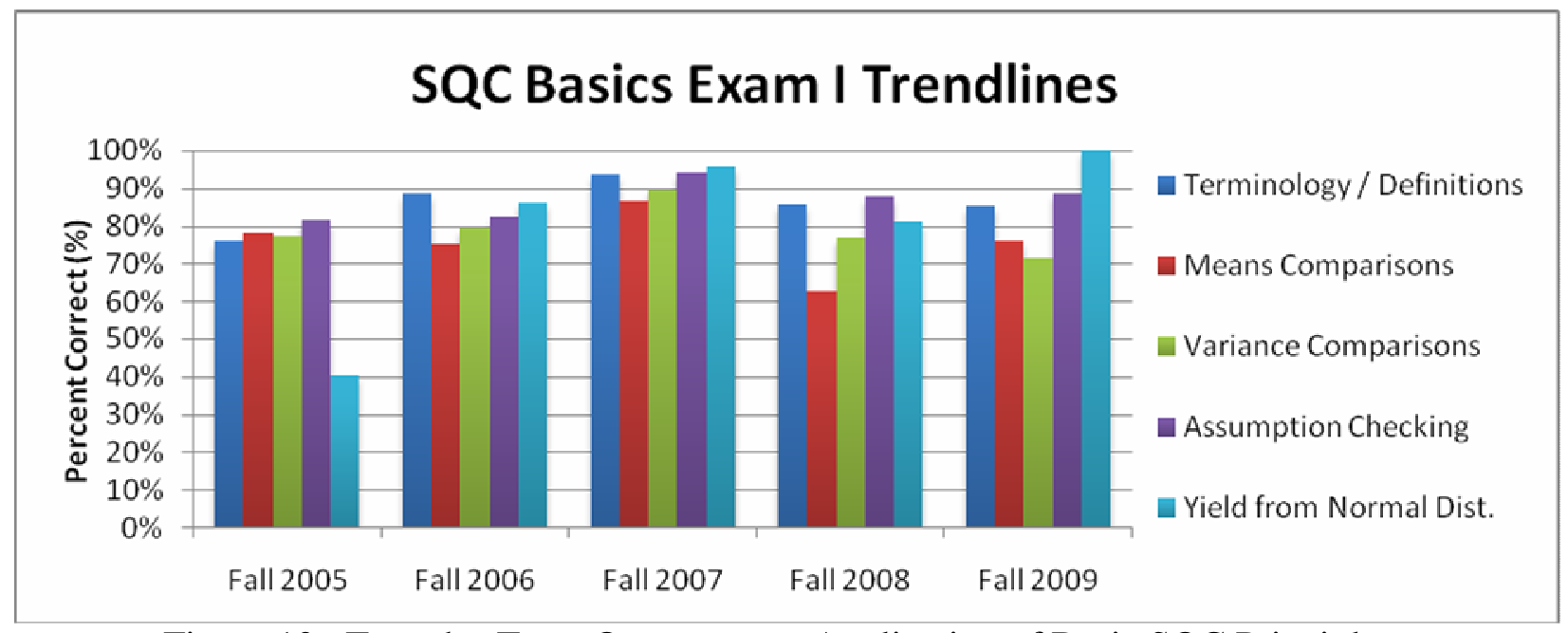

Figure 10. Term-by-Term Outcomes on Application of Basic SQC Principles.

Similar results for continuous variables trendlines and SQC charts and acceptance trendlines are shown in Figures 11 and 12 below. Figure 11 shows gains following incorporation of template simulations and professional journaling with slight erosion of effectiveness in areas when student use of some of these tools were optional. 


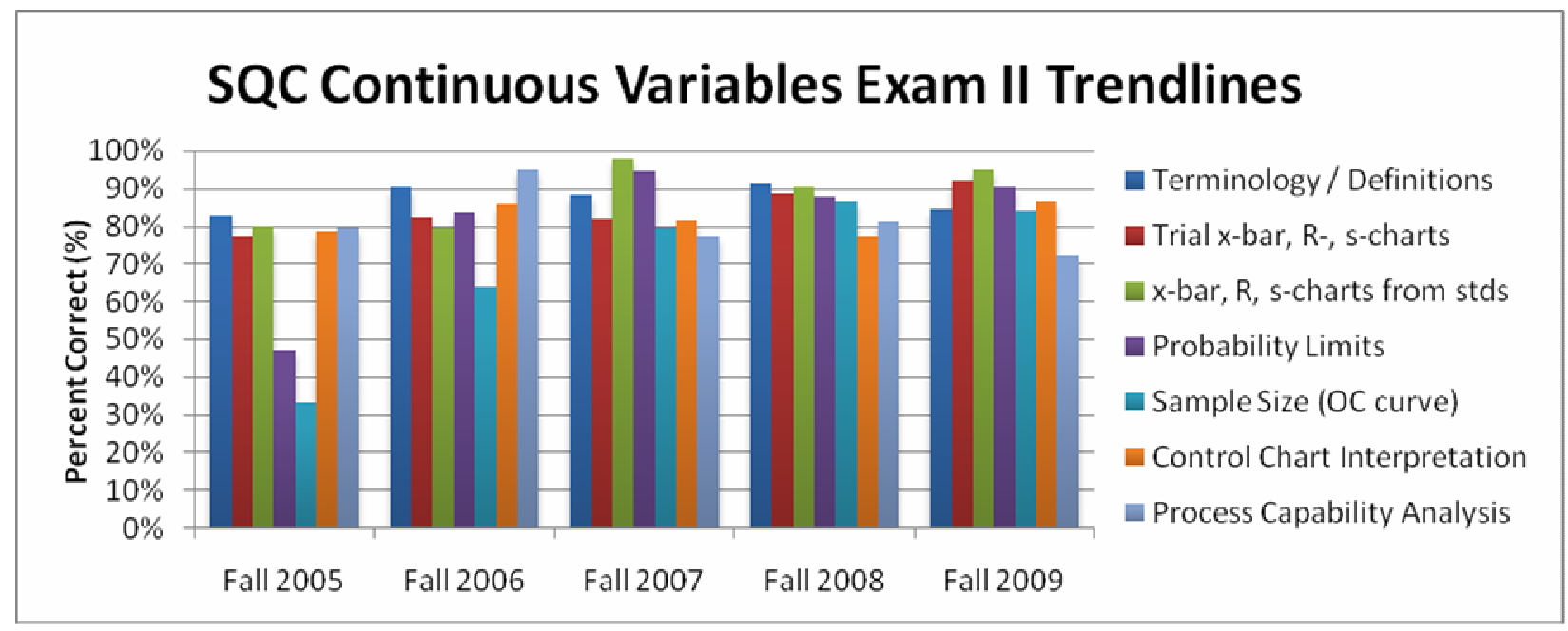

Figure 11. Term-by-Term Outcomes on Application of SQC Continuous Variables

Most of the additional resources for the SQC Individuals Charts and Acceptance Sampling content was implemented in the course from the start (Fall 2005). Variations in aggregate performance (as depicted in Figure 12) tend to occur in a positive direction when the resources were used in-class for assessment (and assignment points), and when used as an in-class development exercise for inclusion in their engineering notebooks.

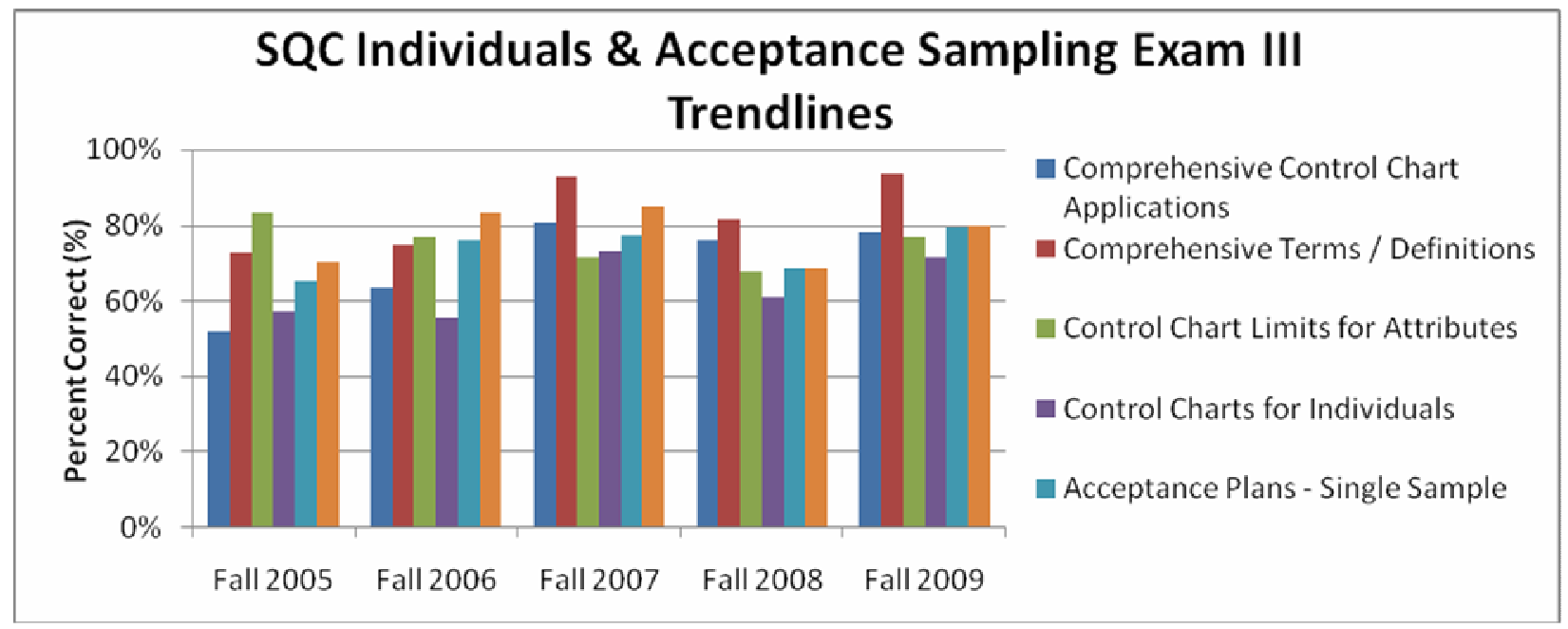

Figure 12. Term-by-Term Outcomes on Application of SQC Acceptance Sampling

Across all three content areas, the detailed data seem to show that: (1) the resources tend to be helpful for those that choose to use it, (2) those that do not choose to use it do quite poorly, and (3) unless there is a substantial incentive (carrot or stick), a good proportion of students will make only a half-hearted attempt to utilize the resources.

\section{The Next Step}

While some gains have been made, it is clear that better strategies for intervention and reinforcement of concepts need to be made. Nevertheless, a number of important lessons have 
been learned over the past years. Interactive modules can provide substantial to aid student understanding but, unless required, students who need the support most are the least likely to take advantage of the support. Student understanding of tougher concepts can be enhanced through interactive modules but require the interaction to be incorporated at the time of the reading. Hands-on experiments and MEAs can be very effective mechanisms for promoting better conceptual understanding, but they do take time and would be more effective if employed when fundamental concepts are first introduced in the introductory courses.

To fully realize the gains that are desired, support modules and classroom activities need to be more formally integrated through an inverted classroom concept. In the traditional classroom, students are assigned a section of a textbook to read before class, though quite a few of them do not. Following a passive reading, if it occurs at all, students attend class where they are passive recipients of a lecture. Following the lecture, students are assigned homework. It is on their own, interacting with their homework, where students first actively engage with the course material. In the inverted classroom, we move the active content engagement from post-class to in-class. The first systematic attempts at classroom inversion came from economics education ${ }^{19}$. In the early attempts, the focus was on creating an environment where "events that have traditionally taken place inside the classroom now take place outside the classroom and vice versa". Thus, the early attempts at inversion moved the passive student engagement with lecture to pre-class by using media, computers, and the internet to provide basic content information to students before class. By providing some content prior to class, the instructor is free to use appropriate active learning methods to draw the students deeper into the material.

\section{References}

1. National Council of Teachers of Mathematics, "Principles and Standards for School Mathematics," Reston, VA: National Council of Teachers of Mathematics, 2000.

2. Streveler, R., Geist, M., Ammerman, R., Sulzbach, C., Miller, R., Olds, B., and Nelson, M., "Identifying and Investigating Difficult Concepts in Engineering Mechanics and Electric Circuits," Proceedings of the American Society for Engineering Education, Chicago, IL, 2006.

3. Miller, R., Streveler, R., Olds, B., Chi, M., Nelson, M., Geist, M., "Misconceptions about Rate Processes: Preliminary Evidence for the Importance of Emergent Conceptual Schemas in Thermal and Transport Sciences," Proceedings of the American Society for Engineering Education, Chicago, IL, 2006.

4. Lessons from Thin Air, Can We Believe Our Eyes, and Private Universe, Produced by the HarvardSmithsonian Center for Astrophysics, 1997. Available from the Annenberg/CPB Math and Science Collection, P.O. Box 2345, South Aburlington, VT 05407.

5. Halloun, I. and Hestenes, D., “The Initial Knowledge State of College Physics Students," American Journal of Physics, 53 (11), ppl 1043-1055, 1985.

6. Hestenes, D., Wells, M., and Swackhamer, G., "Force Concept Inventory," The Physics Teacher, 30, pp. 141158, March 1992.

7. Garfield, J. and Ahlgren, A., "Difficulties in Learning Basic Concepts in Probability and Statistics: Implications for Research, Journal for Research in Mathematics Education, 19 (1), pp. 44-63, 1988.

8. Allen, K., Stone, A., Reed-Rhoads, T., and Murphy, T., “The Statistics Concepts Inventory: Developing a Valid and Reliable Instrument," Proceedings of the American Society for Engineering Education, Salt Lake City, UT, 2004. 
9. Concept Inventory Central, https://engineering.purdue.edu/SCI/workshop/index.html

10. Hake, R., "Interactive-engagement vs. Traditional Methods: A Six-thousdand Student Survey of Mechanics Test Data for Introductory Physics Courses," American Journal of Physics, 6 (1), pp. 64-75, 1998.

11. Smith, K. Sheppard, S., Johnson, D., and Johnson, R., "Pedagogies of Engagement: Classroom-Based Practices," Journal of Engineering Education, 94 (1), pp. 87-102, January 2005.

12. Diefes-Dux, H., Moore, T., Follman, D., Zawajewski, J., and Imbrie, P.K., “A Framework for Posing OpenEnded Engineering Problems: Model Eliciting Activities,”, Savannah GA, 2004.

13. Felder, R.M. and Brent, R., "Understanding Student Differences," Journal of Engineering Education, 94 (1), pp. 87-102, January 2005.

14. Karlin, J. and Kellogg, S., "Metrics and the Holistic Learner," Proceedings of the Research in Engineering Education Symposium (REES), Cairns, Australia, 2009.

15. Lynch, C. L., S. K. Wolcott, and G. E. Huber, "Steps for Better Thinking: A Developmental Problem Solving Process," http://www.WolcottLynch.com, 2002.

16. Diaz, David P. and Ryan B Cartnal, “Students' Learning Styles in Two Classes: Online Distance Learning and Equivalent On-Campus," College Teaching, Vol. 47, no.4, 130-135, 1999.

17. Ross, Jonathan L. and Schultz, Robert A., "Using the World Wide Web to Accommodate Diverse Learning Styles", College Teaching, Vol. 47, no.4, 123-129, 1999.

18. Karlin, J. and Surovek, A, "Come on Down: Multiple Pedagogical Options Using Game Show Formats," Proceedings of the Frontiers in Education Conference, San Antonio, TX, 2009.

19. Lage, M.J., Platt, G. J., and Treglia, M., "Inverting the Classroom: A Gateway to Creating an Inclusive Learning Environment," Journal of Economic Education, 31(1), 30-43, Winter 2000. 